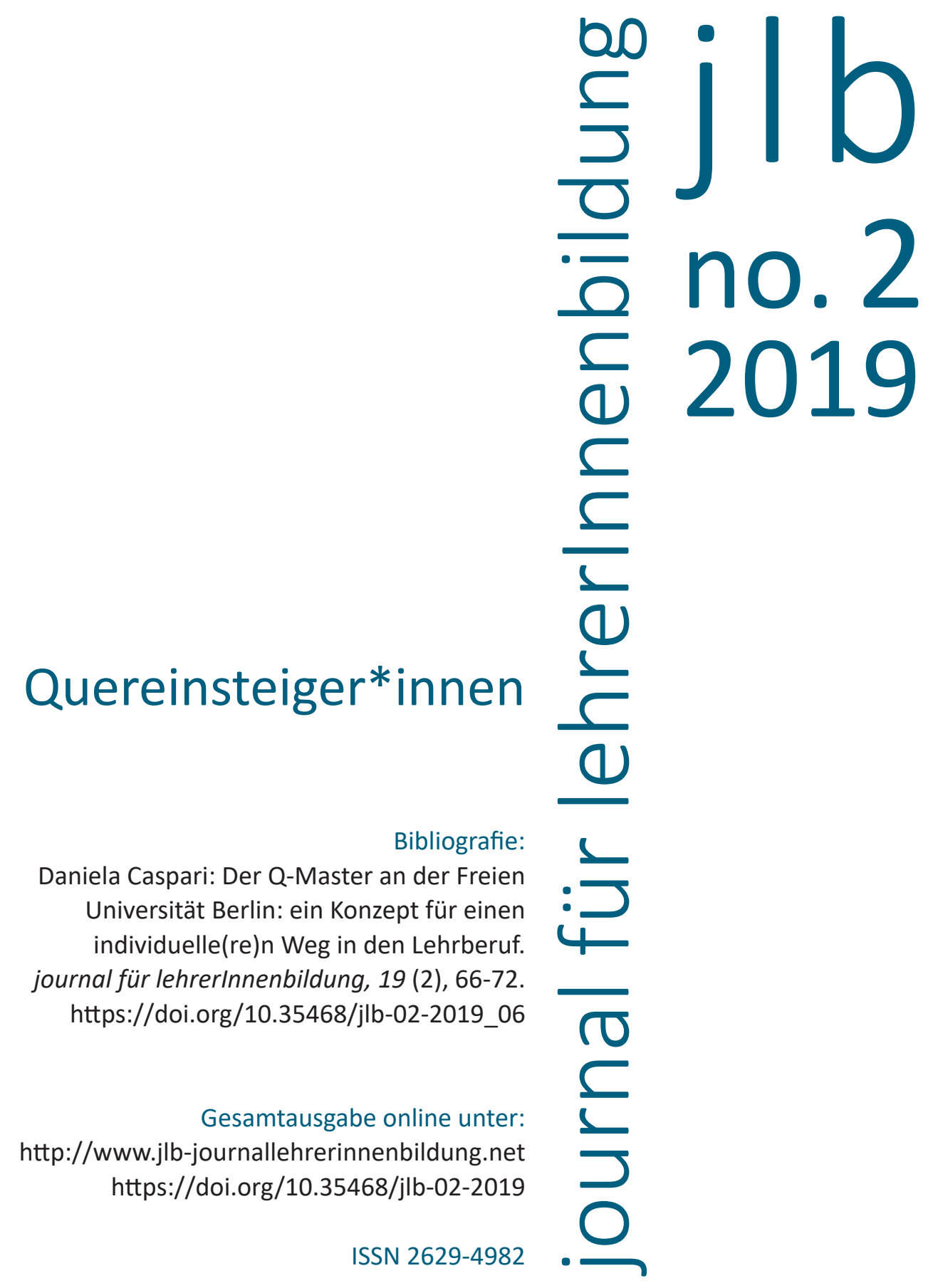


06

Daniela Caspari

\section{Der Q-Master an der Freien Universität Berlin: ein Konzept für einen individuelle(re)n Weg in den Lehrberuf}


Angesichts des Mangels an Lehramtsabsolvent*innen insbesondere in den naturwissenschaftlichen Fächern ergab sich im Rahmen des von der Qualitätsoffensive Lehrerbildung geförderten Projektes K2teach an der Freien Universität die Möglichkeit, in Zusammenarbeit mit der Berliner Senatsverwaltung für Bildung, Jugend und Wissenschaft 2015 einen Studiengang MA of Education mit dem Profil Quereinstieg (kurz: Q-Master) zu entwickeln. Dieser Studiengang wurde vom Land Berlin als Modellversuch genehmigt. Inzwischen hat die 3. Kohorte ihr Studium aufgenommen. Im November 2018 wurde der Modellversuch von der Kultusministerkonferenz (KMK) im Rahmen der "Gestaltung von Sondermaßnahmen zur Gewinnung von Lehrkräften zur Unterrichtsversorgung " unter Punkt 3.2 (,strukturelle Rahmenbedingen“) eingeordnet, das heißt, der Studienabschluss wird auch von anderen Bundesländern anerkannt, sofern dort Bedarf besteht. Der Q-Master wird momentan für die Fächer Informatik, Mathematik, Physik, Englisch, Französisch, Italienisch, Spanisch, Deutsch und Geschichte (die beiden letzteren nur als Zweitfach) angeboten.

Mit dem Q-Master können nun auch solche Bewerber*innen in den MA immatrikuliert werden, die nicht über die in Berlin geforderten Eingangsvoraussetzungen für den Studiengang MA of Education für Gymnasium und Integrierte Sekundarschule verfügen: 90 Leistungspunkte (LP) im 1. Fach, $60 \mathrm{LP}$ im 2. Fach und 30 LP in Erziehungswissenschaften und beiden Fachdidaktiken. Bislang mussten Bewerber*innen trotz großzügiger Anrechnungsregelungen zumeist in den BachelorStudiengang (BA) immatrikuliert werden, um die fehlenden Leistungen zu erwerben. Da die Anrechnungen für die BA-Leistungen auf der Basis der BA-Ordnungen der Freien Universität (FU) erfolgen, führt dies nicht selten dazu, dass die Studierenden nur einen Teil ihrer fachwissenschaftlichen Vorleistungen anerkannt bekommen und sich ihr Studium dadurch weiter verlängert.

\section{Rahmenbedingungen und Ziele}

Beim Q-Master handelt es sich um ein Qualifizierungsangebot für Lehramtsinteressent*innen, die den Studienabschluss MA of Education erwerben möchten, aber nicht die oben genannten Voraussetzungen für die Immatrikulation erfüllen. Als „Blaupause“ wurde die Studienstruktur des Berliner MA of Education-Studiums (inkl. Praxissemester) 
vorgegeben (vgl. Amtsblatt, 2016), auch werden die Anforderungen der KMK-Standards für die Lehrer*innenbildung und die des Berliner Lehrkräftebildungsgesetzes eingehalten. Die Flexibilität in der Studiengestaltung im Q-Master beschränkt sich daher im Wesentlichen auf den Bereich der Fachwissenschaften, d. h. auf insg. 35 LP während des Studiums und 15 LP für die Masterarbeit (insg. 50 LP).

Damit diese Leistungen innerhalb des zweijährigen Masterstudiums erfüllt werden können, wurden als Zugangsvoraussetzungen ein Hochschulabschluss in einem Magister-, Diplom- oder Einfach- bzw. Monobachelorstudiengang festgelegt. Insgesamt müssen in diesem und ggf. weiteren Studiengängen insg. 110 LP in lehramtsrelevanten Fachwissenschaften in beiden Fächern erbracht worden sein, davon mindestens 20 LP im 2. Fach (vgl. Amtsblatt, 2018). Außer dieser im Vergleich zum Regel-Masterstudiengang deutlich flexibleren Zugangsregelung in den Fachwissenschaften werden von den Studierenden keine Studienleistungen in Erziehungswissenschaften und Fachdidaktik erwartet. Dafür verfügen sie über - i. d. R. ausgedehnte - Praxiserfahrungen in pädagogischen Feldern. Darüber hinaus wird die Flexibilität im Vergleich zu Student*innen, die sich wegen fehlender Vorleistungen in den BA immatrikulieren müssen, dadurch erhöht, dass bei der Anrechnung der Studienleistungen für die Zulassung die KMK-Standards als Maßstab dienen und nicht die mitunter doch sehr spezifisch ausgerichteten BA-Module der FU.

Zielgruppe sind Absolvent*innen, die im Rahmen der o.g. Studiengänge idealerweise 90 LP in ihrem ersten Fach und 20 LP in einem zweiten Studienfach erworben haben, z. B. 20 LP Mathematik oder Informatik im Rahmen eines Physik-Monobachelor-Studiums. Mit den für die Fachwissenschaften zur Verfügung stehenden insg. $50 \mathrm{LP}$ absolvieren sie im Rahmen des Q-Masterstudiums das 2. Fach sowie die vorgesehenen Module in Fachdidaktik, Erziehungswissenschaften und Deutsch als Zweitsprache/Sprachbildung. Student*innen der geisteswissenschaftlichen Fächer und Geschichte verfügen i. d. R. über quantitativ ausgewogenere Leistungen in beiden Fächern.

\section{Akzeptanz des Q-Masterstudiengangs}

Obwohl der Q-Master 2016 erst vier Wochen vor Bewerbungsschluss eingerichtet wurde, stieß er direkt auf großes Interesse, das sich in den beiden folgenden Jahren weiter vergrößert hat. 
Kohorte 1: Beginn WS 2016/2017

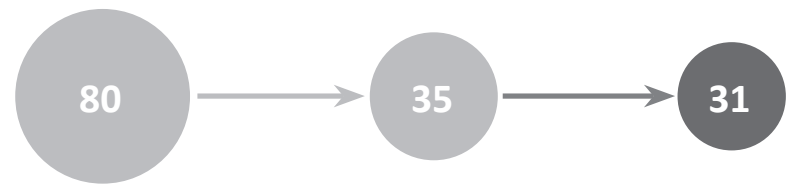

Kohorte 2: Beginn WS 2017/2018

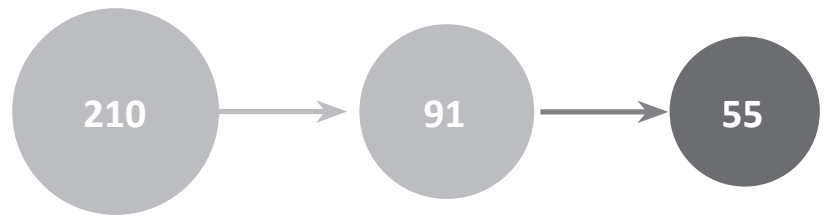

Kohorte 3: Beginn WS 2018/2019

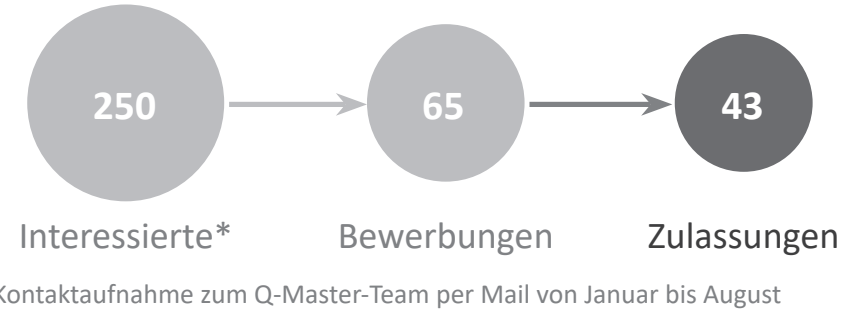

Abb. 1 Entwicklung der Kohorten

Dabei verteilen sich die Studierenden wie folgt auf die Fächer:

Kohorte 1, Kohorte 2 und Kohorte $3(n=129)$

\begin{tabular}{|c|c|c|c|c|c|c|c|c|c|c|}
\hline $\begin{array}{l}\text { Zweitfach } \\
\text { Erstfach }\end{array}$ & Eng & Frz & Info & Ita & Ma & $\mathrm{Ph}$ & Spa & Deu & $\mathrm{Ge}$ & $\Sigma$ \\
\hline Englisch & - & 1 & 3 & - & - & - & 5 & 18 & 10 & 37 \\
\hline Französisch & - & - & - & 1 & - & - & - & 6 & 3 & 10 \\
\hline Informatik & - & - & - & - & 16 & 1 & - & - & - & 17 \\
\hline Italienisch & 1 & 2 & - & - & - & - & 1 & 4 & 2 & 10 \\
\hline Mathematik & 1 & - & 17 & - & - & 7 & - & - & 1 & 26 \\
\hline Physik & - & - & - & - & 16 & - & - & - & - & 16 \\
\hline Spanisch & - & 1 & - & - & - & 1 & - & 5 & 6 & 13 \\
\hline$\Sigma$ & 2 & 4 & 20 & 1 & 32 & 9 & 6 & 33 & 22 & 129 \\
\hline
\end{tabular}

Abb. 2 Gewählte Fächerkombinationen 
Bislang, d. h. fünf Semester nach Einrichtung des Studiengangs, haben 16 Student*innen der 1. Kohorte den Q-Master erfolgreich abgeschlossen, die 2. Kohorte hat gerade das Praxissemester, die 3. Kohorte das 1. Semester beendet. Aus der 1. Kohorte haben sieben Studierende das Studium vorzeitig abgebrochen. Zwei Studierende, einer aus der 1. und einer aus der 2. Kohorte, haben sich dabei für das berufsbegleitende Referendariat (Quereinstiegsprogramm der Senatsverwaltung) entschieden, wobei der zweite jedoch in den Q-Master zurückgekehrt ist. Dass noch nicht alle Studierenden der 1. Kohorte ihr Studium in Regelstudienzeit beendet haben, liegt vor allem daran, dass die meisten parallel zum Studium für ihren Lebensunterhalt arbeiten müssen und häufig Kinder haben.

\section{Erfahrungen mit dem Q-Master}

\section{Anzahl der Zulassungen}

Die hohe "Schwundquote" zwischen der Anzahl der Interessent*innen und der der Immatrikulierten ergibt sich daraus, dass viele nicht über die passenden Abschlüsse verfügen. In den MINT-Fächern erfüllen i. d. R. nur Studierende aus Diplom- oder reinen Mono-Bachelorstudiengängen die Voraussetzungen, nicht jedoch z. B. Absolvent*innen der Ingenieurswissenschaften, der Geowissenschaften oder der Meteorologie. In den sprachlichen Fächern gibt es häufig Anfragen aus Kombioder 2-Fach-Bachelor-Studiengängen, die jedoch laut Zugangssatzung ausgeschlossen sind. Absolvent*innen der Übersetzungswissenschaft haben i. d. R. nicht genügend philologische Anteile absolviert.

\section{Motivation}

Aus den Eingangsbefragungen wird deutlich, dass sich die Berufswahlmotivation der Q-Master-Student*innen nicht von denen der Regel-Lehramtsstudent*innen unterscheidet. Viele verfügen bereits über pädagogische Erfahrung und wünschen sich das für den Lehrberuf notwendige Professionswissen. Die meisten erhoffen sich eine fundierte Ausbildung, insb. in Form von Grundlagenwissen in den Erziehungswissenschaften und den Fachdidaktiken. Studierende, die parallel bereits in der Schule tätig sind, erhoffen sich dagegen oft anwendungsbezogenes Wissen und praxisbezogenen Erfahrungsaustausch. 


\section{Zufriedenheit}

Die Zwischenfeedbacks und die Abschlussbefragung zeigen, dass die Student*innen mit dem Q-Master durchwegs zufrieden bis sehr zufrieden sind. Sie schätzen die Möglichkeit, in überschaubarer Zeit einen anerkannten Hochschulabschluss sowie eine fundierte Vorbereitung auf das Referendariat zu erhalten. Dabei heben sie das Praxissemester hervor, in dem sie die im Vergleich zum berufsbegleitenden Referendariat eine komplexitätsreduzierte Form von Praxiserfahrung erleben. Sie schätzen die kombinierte Begleitung aus Schule und Hochschule und erleben das im Q-Master erworbene Wissen als hilfreich für die Planung, Durchführung und Reflexion von Unterricht.

\section{Beratung}

Der Q-Master ist ein extrem beratungsintensiver Studiengang. Bereits die Sichtung und Anrechnung der mitgebrachten Studienleistungen ist sehr zeitaufwändig, insbesondere bei ausländischen Studienabschlüssen. Dasselbe gilt für die Erstellung der individuellen Studienverlaufspläne. Zusätzlich erhalten alle Studierenden am Ende des 2. Semesters eine Zwischen- und vor Studienende eine Abschlussberatung. Dadurch stellen wir sicher, dass trotz der individualisierten Verlaufspläne alle für den Abschluss notwendigen Leistungen erbracht werden.

\section{Fazit und Ausblick}

Trotz des hohen Aufwandes, den wir im weiteren Projektverlauf noch stärker zu reduzieren suchen, ist der Q-Master ein Gewinn: Ein Gewinn für die Student*innen, die ihre Dankbarkeit für diese Qualifizierungsmöglichkeit oft deutlich äußern. Er ist aber auch ein Gewinn für die Freie Universität, die dadurch gerade in Mangelfächern die Zahl der Lehramtsstudent*innen erhöhen konnte, und für die Berliner Schule, weil nur der kleinere Teil der Q-Master-Student*innen für den von ihnen angebotenen Quereinstieg in Frage gekommen wäre. Darüber hinaus bietet der Q-Master ein Konzept, wie man den zunehmend individualisierten Lebensläufen von Lehramtsinteressent*innen besser gerecht werden kann, ohne Abstriche an der Qualität der Ausbildung zu machen (vgl. hierzu auch Caspari, 2018 sowie das Positions- 
papier der GFD, 2018). Die abschließende Evaluation des Q-Masters steht zwar noch aus, aber er scheint so viel Potenzial zu besitzen, dass wir in der 2. Projektphase die Einrichtung eines Q-Masters für Absolvent*innen mit einem Kombi-Bachelor-Abschluss planen.

\section{Literatur}

Amtsblatt der Freien Universität (2016). Studien- und Prüfungsordnung der Freien Universität Berlin für den Masterstudiengang für ein Lehramt an Gymnasien mit dem Profil Quereinstieg. Verfügbar unter https://www.fu-berlin.de/service/ zuvdocs/amtsblatt/2016/ab342016.pdf [07.08.2019].

Amtsblatt der Freien Universität (2018). Zugangssatzung der Freien Universität Berlin für den Masterstudiengang für ein Lehramt an Gymnasien mit dem Profil Quereinstieg. Verfügbar unter https://www.fu-berlin.de/service/zuvdocs/amtsblatt/ 2018/ab222018.pdf [15.03.2019].

Caspari, D. (2018). Zur Professionalisierung von Fremdsprachenlehrpersonen Überlegungen im Kontext von Quer- und Seiteneinstieg. In E. Burwitz-Melzer, F. G. Königs, C. Riemer \& L. Schmelter (Hrsg.), Rolle und Professionalität von Fremdsprachenlehrpersonen. Inklusion, Diversität und das Lehren und Lernen fremder Sprachen (S. 32-42). Tübingen: Narr.

Gesellschaft für Fachdidaktiken (GFD) (2018). Ergänzende Wege der Professionalisierung von Lehrkräften Positionspapier der GFD zur Problematik des Quer- und Seiteneinstiegs. Verfügbar unter http://www.fachdidaktik.org/veroeffentlichungen/ positionspapiere-der-gfd [15.03.2019].

Weitere Informationen zum Q-Master finden Sie unter:

https://www.fu-berlin.de/sites/k2teach/foerderphase1/tp4/index.html [07.08.2019]

https://www.fu-berlin.de/sites/dse/master/qmaster/index.html [15.03.2019]

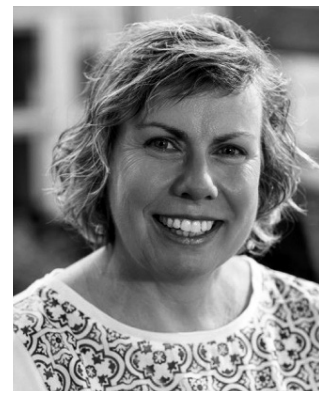

Daniela Caspari, Dr., Prof.

für Didaktik der Romanischen Sprachen

an der Freien Universität Berlin.

Arbeitsschwerpunkte:

Lehrer*innenforschung,

kompetenzorientierter Fremdsprachenunterricht,

Literaturdidaktik

caspari@zedat.fu-berlin.de 